\title{
IDENTIDAD Y RELIGIÓN EN INMIGRACIÓN. A PROPÓSITO DE LAS ESTRATEGIAS DE INSERCIÓN DE LOS MUSULMANES SENEGALESES
}

\section{JOAN LACOMBA}

Departament de Treball Social. Universitat de València.

\section{INTRODUCCIÓN}

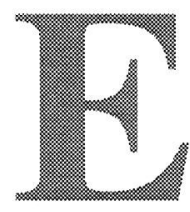

1 estudio de la compleja relación que se establece entre la dimensión religiosa y la dimensión identitaria a propósito de la experiencia migratoria de la población musulmana corre el riesgo de atribuir características genéricas a un colectivo que presenta una importante heterogeneidad en su interior, pues en el proceso de integración de la inmigración musulmana, la población aparece socialmente estratificada. No es homogénea y no sigue una trayectoria unívoca en la sociedad (F. Dassetto, 1995: 103).

Igualmente podemos caer en la adjudicación de un desproporcionado peso a la religión frente a otros factores que juegan un papel no menos importante en los procesos de estructuración y reconstrucción identitaria de los inmigrantes, tanto musulmanes como no musulmanes ${ }^{1}$.

Es por ello necesario comenzar por puntualizar que la religión constituye, en el caso de determinados colectivos migrantes minoritarios en los países de destino (el caso de la inmigración musulmana, pero también el de otros colectivos actualmente o en un pasado reciente: inmigración judía en la diáspora, católicos italianos en los Estados Unidos,...), un pilar central en la organización de las modalidades de inserción y negociación con la nueva sociedad, sin que ello sea una característica exclusiva del colectivo que nos ocupa aquí.

Además, junto a la religión (enmarcable siempre dentro de una cultura de referencia más amplia de la que no puede desgajarse) otras variables básicas han de tenerse en cuenta para comprender la multiplicidad de estrategias y respuestas ante el reto migratorio. El sexo, la

1 Sobre los factores culturales en la inserción de los inmigrantes musulmanes de una forma genérica pueden ampliarse las tesis en J. Lacomba, 1996. 
edad, la nacionalidad, la etnia, el ámbito de origen (rural o urbano), el nivel de estudios y formación, el conocimiento de lenguas, la trayectoria migratoria o el tiempo de residencia, son algunas de ellas. Es en función de la combinación de esas variables como podrán dibujarse diferentes tipos de estructuración de la relación identidad/religión, al poner en evidencia la variedad de estrategias vitales que determinan la diversidad de modalidades de inserción para cada uno de los grupos e individuos. Igualmente, y en base a esas mismas variables, habría que destacar la capacidad de gestación por los actores de identidades múltiples que se ponen en juego en la vida cotidiana.

\section{INMIGRACIÓN: IDENTIDAD Y RELIGIÓN}

\subsection{Identidad y pertenencia: los grupos étnicos}

El término grupo étnico no es de fácil conceptualización. Marco Martiniello reconoce que pueden distinguirse dos grandes tipos de aproximaciones al fenómeno de la etnicidad en la sociedad contemporánea. En primer lugar, la aproximación sustancialista o primordialista trata la etnicidad como un dato objetivo, inmutable e incontestable inscrito en la filiación de los grupos humanos y transmitiéndose de alguna manera por la sangre. Esta aproximación tiende a reificar los grupos étnicos y a atribuir a simples categorías una realidad que no presentan necesariamente. En segundo lugar, la aproximación subjetivista y constructivista considera la etnicidad y los grupos étnicos como construcciones sociales que no exigen necesariamente la existencia objetiva de rasgos substanciales distintivos para las etnias. De acuerdo con Max Weber, Martiniello sustenta que la creencia subjetiva en una comunidad de origen fundada en una similitud cultural, de costumbres y de experiencia basta para dar lugar a un grupo étnico, cualquiera que sea la verdad objetiva de estas similitudes (M. Martiniello, 1994: 106107).

Martiniello insiste en el carácter socialmente construido de los grupos étnicos y de la categoría étnica como una noción abstracta elaborada por el investigador sobre la base de una selección de criterios objetivos que caracterizan la población de origen inmigrado. Desde este punto de vista los trazos objetivos que permiten caracterizar la categoría étnica son a menudo socialmente percibidos como criterios diferenciadores válidos y validables de un conjunto humano sobre los que desarrollar y reproducir imágenes positivas o negativas hasta su conversión en prejuicios más o menos estables y sólidos. La noción de categoría étnica es pues, a la vez, producto de una construcción sociológica y de una construcción social (M. Martiniello, 1994: 107). 
La identidad étnica de un individuo como adscripción a una categoría étnica no resulta de una decisión que repose sobre una identificación sino más bien de un proceso de clasificación que le es exterior. Sin embargo, el concepto de pertenencia ayuda a explicar el hecho de que los individuos étnicamente categorizados puedan desarrollar un sentimiento de pertenencia a un mismo grupo, y que ello les pueda permitir, por ejemplo, crear redes de solidaridad o una red de instituciones y de asociaciones destinadas a defender sus intereses (M. Martiniello, 1994: 107).

En este sentido, y uniendo ambos conceptos, puede decirse que la identidad étnica es una clase de sentimiento de pertenencia que vincula al individuo con la colectividad a la que pertenece por adscripción. Según Joan Josep Pujadas «un sentimiento que se sustenta en todo un conjunto de valores, acciones y símbolos que constituyen un campo de comunicación y de interacción entre los individuos de una misma colectividad y que, al mismo tiempo, actúan como factores de diferenciación respecto a otros grupos». Por ello, escribe el mismo Pujadas, «la experiencia migratoria, que sitúa a los individuos fuera de su grupo y los pone en contacto con otros universos simbólicos, proporciona la oportunidad de estudiar las funciones adaptativas de la identidad, las bases sociales en que su fundamenta y el proceso de redefinición de aquellos valores, acciones y símbolos que poseen relevancia y significación emotiva para los actores sociales» (J.J. Pujadas y D. Comas, 1991: 35).

Pero, más allá de su componente étnico, en el ámbito de los estudios migratorios, la identidad ha de ser conceptualizada en un marco más amplio que muestre la riqueza de los juegos y las estrategias que pueden desarrollarse por una y otra parte: «La identidad del individuo es abusivamente reducida al origen étnico de éste. Se supone que la identidad individual estaría construida por la pertenencia a uno o unos grupos étnicos, mientras que ésta es construida a través de esa pertenencia así como a través de las relaciones con otras identidades colectivas de orden diferente: sexo, clase social, confesión religiosa,..., por el individuo que les da sentido interpretándolas» (M. Giraud, 1993: 237).

La identidad, como venimos manteniendo, es un fenómeno que surge de la dialéctica entre el individuo y la sociedad (P. Berger y $\mathrm{T}$. Luckmann, 1968: 65). La noción de estrategia identitaria nos ayudará a definir con mayor precisión el carácter instrumental de la identidad, entendiendo por estrategia «los procedimientos puestos en práctica (de forma consciente o inconsciente) por un actor (individual o colectivo) para alcanzar una o unas finalidades (definidas explícitamente o situándose al nivel del inconsciente) y elaborados en función de la situación de interacción, es decir, en función de las diferentes determina- 
ciones (socio-históricas, culturales, psicológicas) de la misma» (C. Camilleri, 1990: 24).

Las estrategias de identificación construidas por los individuos en sus interacciones recubren la noción de identidad cultural y conforman los procedimientos por los cuales los actores sociales se coaligan y se distinguen, se constituyen para la acción en grupo, exponiendo una pertenencia étnica (M. Giraud, 1993: 238).

«Desde una perspectiva dinámica, me parece preferible considerar la cultura como un depósito de prácticas más bien que como un conjunto de prescripciones fijas, dicho de otro modo, como un espacio en el que se despliegan las estrategias de los actores sociales, con el resultado de la producción de figuras compuestas, híbridas, sincréticas del individuo y su identidad. Es en términos de lógicas mestizas, de construcción negociada, como hay que aprehender la empresa de deconstrucción/reconstrucción identitaria. Una identidad con geometría variable que se manifiesta en una multiplicidad de elementos entre los cuales la referencia étnica no es más que un componente (lengua, creencias religiosas, status social,...)» (J.F. Werner, 1993: 277).

\subsection{Religión, comunidad y solidaridad}

Una larga serie de diferentes estudios sobre el rol de la religión en el proceso de construcción identitaria y de pertenencia colectiva de las comunidades inmigradas musulmanas muestran que la comunidad religiosa constituye no sólo la estructura de identificación social diferenciada, sino que puede convertirse en realidad en la llave de la inserción en la sociedad de acogida, determinando en gran medida las estrategias de inserción social ${ }^{2}$.

En la sociedad moderna la comunidad no goza de una buena acogi$\mathrm{da}^{3}$. Lo mismo podría decirse de la religión. Sin embargo, el fenómeno migratorio (en especial en su versión musulmana) y otros, como los llamados «movimientos socio-religiosos», han puesto de actualidad la cuestión de la comunidad y la religión como elementos de regulación en sociedades que viven una crisis continuada: «Ciertas sociedades europeas, en grados diversos, han relegado lo religioso a la esfera individual y el espacio privado. La inmigración, al acentuar la aproximación comunitaria y el derecho a la manifestación colectiva y pública de su religión, ha planteado la cuestión del retorno de lo religioso como

2 J. Cesari, 1994; F. Dassetto, 1994; B. Etienne, 1989; G. Kepel, 1987; R. Leveau, 1988; B. Lewis y D. Schnapper, 1992.

3 En los últimos años se ha desarrollado en el campo de la teoría sociológica, con resultados diversos y trayectorias irregulares, una corriente llamada "comunitarista" que trata de hacer de contrapeso al individualismo predominante en las ciencias sociales contemporáneas. 
un retorno de lo desplazado. Retorno que no se identifica necesariamente con las prácticas religiosas (que pueden incluso retroceder), sino que expresa un deseo de reconocimiento de identidad social y cultural. Los inmigrados, excluidos de las formas tradicionales de representación política, hacen así posible, en nombre de los derechos del hombre y del respeto de los principios democráticos que rechazan toda exclusión o marginación, el retorno de lo religioso en el espacio social, antaño marginalizado a la esfera privada por los procesos de laicización y secularización. Lo religioso, en ciertas circunstancias, puede jugar un rol transitorio o permanente susceptible de ofrecer al individuo aislado estructuras intermedias de integración y de lucha contra la marginación» (A. Perotti, 1994: 5).

Nos advierte en el mismo sentido Dominique Schnapper que hay que evitar ver en la conservación de una comunidad concreta tan sólo un factor de conservación de las formas tradicionales de la vida social, pues, del mismo modo que la familia, «la comunidad desempeña un papel conservador, pero también proporciona al emigrante una forma de integración en la sociedad receptora, ayudándole a resolver los conflictos con la sociedad global, favoreciendo la reducción de los conflictos familiares y la elaboración de una aculturación limitada» (D. Schnapper, 1988: 184).

Frente a la adversidad la comunidad permite tejer y reforzar los lazos de solidaridad: "La solidaridad y la ayuda mutua constituyen la base de la sobrevivencia, mitigan el traumatismo de la llegada a un nuevo contexto social y posibilitan la adaptación. La reconstrucción de antiguos lazos sociales en un nuevo contexto resulta así la condición misma para la reproducción del grupo doméstico y, de forma general, para la inserción social de los emigrantes» (J.J. Pujadas y D. Comas, 1991: 38).

EI repliegue comunitario ante una sociedad que ofrece reducidas posibilidades de inserción, es decir, que favorece la heteroexclusión, constituye una potente estrategia de adaptación al medio: «Las situaciones de precariedad económica y de crisis reproductiva de los grupos domésticos nucleares generan una multiplicidad de respuestas organizativas. Para sobrevivir, la gente trasplanta las antiguas reglas de solidaridad, reinterpretándolas y creando a partir de ellas múltiples formas de entreayuda y de cohesión. Y es que cuando hay que enfrentarse a una forma de vida nueva, a relaciones sociales que no se dominan, a unos códigos lingüísticos y de comportamiento que resultan extraños, la red de parientes o paisanos procura una identificación social que ni la residencia ni el trabajo proporcionan por sí solas: dan sentido de estabilidad, de pertenencia a un grupo, funcionan, de hecho, como una protección del individuo ante un entorno social nuevo, desconocido y potencialmente hostil para él, pues contribuyen a crear un 
microuniverso en el que se ejerce la ayuda y la solidaridad, en el que se controlan las relaciones, en el que se pueden reproducir las pautas de comportamiento propias» (J.J. Pujadas y D. Comas, 1991: 53-54).

Hemos visto que, en términos generales, puede decirse que un grupo étnico es un grupo humano con características culturales y sociales propias atribuidas en un proceso de construcción social. Sin embargo, cuando un grupo étnico está en una posición de subordinación en relación con la mayoría y, sobre todo, cuando él mismo se asegura su propia reproducción en tanto que grupo, sus propios servicios sociales y su propia subsistencia, basándose esencialmente sobre la etnicidad, se corre el riesgo de convertirlo en minoría étnica. Con su actitud la sociedad de acogida puede transformar al grupo étnico en minoría étnica o minoría marginada, replegada sobre sí misma, reducida a preservar en lo fundamental su cultura y su religión (C. Giménez, 1995: 281).

\subsection{Islam: identidad religiosa en inmigración}

El análisis de la relación entre religión e identidad a propósito de la inmigración musulmana plantea problemas tan básicos (y a menudo tan obviados) como el de la propia definición del inmigrante musulmán. Con frecuencia se comete un grave error cuando se presupone la pertenencia religiosa a partir de la procedencia nacional. La única posibilidad de escapar a esa tendencia es «responder a la cuestión a partir del parámetro individual (subjetivo) de la autodefinición, diciendo: es musulmán el que se considera como tal. Esta definición mínima supone una libertad de conciencia y de práctica. Pues el Islam es maneras de ser, maneras de pensar, de obrar, producidas, transmitidas e interiorizadas por los individuos en grupos y sociedades diferentes. Pero también es modo de contestación o de legitimación, de integración o de marginación según los individuos y de su posición en una sociedad» (M. Belbah, 1994: 331-332).

Insistiendo en la heterogeneidad y estratificación de los inmigrantes Belbah añade que se trata de individuos que negocian cuadros de vida, condiciones de existencia tanto materiales como simbólicas. Cada uno conduce esta negociación en función de los atributos de que dispone y de los objetivos que se da (M. Belbah, 1994: 344).

Es más, ni siquiera aquellos que se consideran como tales musulmanes, lo entienden o lo hacen del mismo modo. De hecho, las poblaciones musulmanas pueden ser diferenciadas en cuanto a la referencia al Islam. Felice Dassetto emplea una clasificación en la que coloca en un extremo los agnósticos declarados y, en el otro extremo, todos los creyentes que afirman su pertenencia al Islam. Entre esos dos extremos se situarían los que viven su pertenencia al Islam como cultura, pero no se disocian, o poco, de los que definen su pertenencia en térmi- 
nos explícitamente religiosos. Por último, en el grupo de los religiosos, se puede distinguir un Islam doméstico de tipo privado, de un discurso de tipo ideológico ligado a las formas de Islam organizado o militante (F. Dassetto, 1995: 104).

Es posible desde esta perspectiva construir una tipología de las formas de pertenencia religiosa musulmana:

- pertenencia negativa: agnósticos, indiferentes.

- pertenencia culturalista: élites occidentalizadas, jóvenes segunda generación.

- pertenencia ritualista: devocionalismo privado.

- pertenencia de participación: devocionalismo público.

- pertenencia organizada militante/política: islamistas.

- pertenencia fronteriza: marabustismo (cofradías) y sufismo.

Esas diferentes formas de pertenencia no son definitivas, son móviles y pueden superponerse entre ellas en función de los procesos de desestructuración y reestructuración identitaria.

Asimismo, y para completar el contenido de la tipología, sería necesario el estudio de las prácticas islámicas (oración, ayuno, peregrinación, prácticas devocionales o festivas, prohibiciones alimenticias,...) y su significación como indicadores de la pertenencia al Islam. Sin olvidar la importancia de los ritos de paso ligados al ciclo vital (circuncisión, boda, entierro,...) en el contexto migratorio, al contribuir al mantenimiento y a la reproducción de la cohesión social, pues son momentos de enseñanza durante los cuales la sociedad trata de integrar a los individuos en su seno y de generar sentimientos que sirvan de guía en la vida social (F. Reysoo, 1991: 11).

\section{LA INMIGRACIÓN MUSULMANA SENEGALESA}

La inmigración musulmana senegalesa ofrece una serie de particularidades, incluso frente a otros colectivos inmigrantes en los que es mayoritaria la misma religión, que la convierten en un terreno privilegiado para el estudio de la relación existente entre la dimensión identitaria religiosa y las estrategias de inserción. Son precisamente esas características específicas las que trataremos de desarrollar y analizar aquí.

A su vez, la complejidad étnico-lingüística y religiosa de Senegal es notable y poco conocida. Sin embargo, su estudio es imprescindible para comprender las variables que operan en la configuración de las dinámicas y procesos que condicionan en gran medida las propias estrategias de inserción de los inmigrantes senegaleses en nuestra sociedad. 


\subsection{Las cofradias y el Islam murid}

La importancia del fenómeno cofrádico, tal y como ahora lo definiremos, reside en el hecho de que el Islam en su lectura murid constituye la cultura de pertenencia de una parte importante de la población senegalesa relacionada de forma directa o indirecta con la inmigración en nuestro país. Se calcula que en 1989 el 94\% de la población de Senegal era de religión musulmana y que más de $3 / 4$ partes de la misma pertenecía a alguna de las tres cofradías mayoritarias (un $49 \%$ a la cofradía tijaniya, un $33 \%$ a la muridiya y un $12 \%$ a la qadiriya) (O. Schmidt, 1994: 11).

La importancia social de las cofradías en Senegal queda puesta de manifiesto en las observaciones del investigador senegalés Mustafá Anta Diop: «Minoría dentro de otra minoría, los musulmanes negroafricanos -sobre todo los senegaleses- ofrecen trazos bien específicos en cuanto a sus instituciones religiosas y a sus prácticas. Los adeptos de estos grupos religiosos (cofradías) mantienen con su líder espiritual respectivo relaciones complejas basadas en el sistema de donaciones y contra-donaciones. Las cofradías se estructuran en dahiras (círculos religiosos). Las reuniones semanales giran en torno a cuestiones de orden teológico, cantos religiosos o sesiones de lectura del Corán. El sistema de dahira estructura la vida social de sus miembros, tomando a cargo las diferentes ceremonias de la vida: nacimiento, atribución de nombre, boda, defunción,...» (M.A. Diop, 1990: 78-79).

Según las palabras de Gilles Kepel «la cofradía tijaniya es una de las más importantes órdenes místicas musulmanas en África del Norte y del Oeste. Fue fundada en 1789 por Ahmed El Tijani, nacido hacia 1737 en la población marroquí de Ain Madi, y persigue garantizar a sus adeptos la salud en el más allá, estableciendo entre ellos un cierto número de relaciones y redes sociales de solidaridad. Los tijan se distinguen de otros musulmanes, en general, y de otros grupos místicos o sufíes, en particular, en el cumplimiento de un cierto número de ritos que les son propios -sobre todo la recitación de letanías y oraciones específicas-. Si la rama argelina de la cofradía ha sido uno de los más firmes sostenes de la colonización francesa en ese país, en África del Oeste ha constituido uno de los instrumentos más vigorosos de la islamización de las poblaciones animistas, y está particularmente bien implantada entre los soninkés, que forman el grueso de la inmigración musulmana africana en Francia» (G. Kepel, 1987: 130).

Por su parte, la etnia wolof (etnia mayoritaria a la que pertenece el grueso de la inmigración senegalesa en España, y especialmente en Valencia ${ }^{4}$ ) constituye el núcleo de los efectivos de la cofradía murid

4 Entre 1994 y 95 realizamos, con la financiación de la Institució Valenciana d'Estudis i 
que, por otra parte, ha venido ganando terreno en los últimos años en detrimento cada vez más de las otras cofradías. Ciertamente, en el caso senegalés el Islam de las cofradías ha tenido una de sus expresiones más exitosas en el movimiento murid, fundado en la época colonial por el cheik Ahmadou Bamba y convertido en fuerza de resistencia al dominio francés.

En cuanto a su origen, el fenómeno de las cofradías procede del desarrollo del misticismo sufí en África del Norte. Ampliamente extendido en el Magreb, y sobre todo en Marruecos, es igualmente de esta región de donde proviene la islamización de las zonas sudanesas, entre las cuales se encuentra Senegal (J. Copans, 1980: 164).

Por ejemplo, el marabutismo o culto a los santos es una forma de religiosidad particularmente extendida en Marruecos, en donde el recurso a los santos tiene principalmente un objetivo terapéutico $y$, en menor medida, fines psicológico-afectivos. Los medios utilizados mezclan los efectos catárticos, la utilización de la farmacopea tradicional, la brujería y la adivinación. El recurso a los santos puede explicarse por su función mediadora entre Dios y los humanos, pero también por la falta de soluciones sociales a los problemas sanitarios y societarios. Un cierto renombre, la amplificación publicitaria de los éxitos y las virtudes de un santo acrecientan considerablemente el número de adeptos. En general, el éxito de los santos puede comprenderse en el contexto de una modernización desestructurante e inacabada (F. Dassetto, 1984: 44).

Según Felice Dassetto ${ }^{5}$ los términos generales de cofradía y cofraderismo son utilizados para designar las formas de asociación religiosa, en la frontera del Islam ortodoxo, que se caracterizan por una organización, una creencia y un ritual específicos. Están fundadas por un maestro (cheik) que predica a sus discípulos una vía mística, un camino hacia dios (tariqa) que los miembros de la cofradía aceptan y siguen. Se trataría de un conjunto de actores sociales reunidos según status en una red coordinada de funciones que atribuyen a cada uno tareas y responsabilidades específicas. Como toda organización las tariqas tienen sus normas y sus sistema de sanciones y de promoción,

Investigacions, un estudio sobre las condiciones de vida de los inmigrantes marroquíes y senegaleses en el área de Valencia que permitió corroborar el carácter homogéneamente étnico de la comunidad senegalesa (el total de los entrevistados era de etnia wolof) y un alto porcentaje de pertenencia a la cofradía musulmana murid. Véase J. Lacomba, 1995

5 El profesor Felice Dassetto ha sido pionero en el estudio del fenómeno de las cofradías y otras formas de religiosidad no ortodoxas entre la población inmigrante musulınana en Bélgica, como el caso de la extensión del movimiento Tabligh entre los marroquíes. Puede consultarse al respecto $F$. Dassetto, 1988. En el caso de Estado español la influencia del Tabligh puede rastrearse en B. Aguer, 1991. 
así como un modo particular de lenguaje y de comunicación (F. Dassetto, 1984: 114-118).

La tariqa (vía, método) se ha revelado una respuesta particularmente fértil para una minoría en busca de una forma de religiosidad vuelta hacia la meditación y la práctica ascética. De otro lado, el culto del santo, la obediencia al guía espiritual (marabut) y la inserción en la práctica mística de elementos no islámicos, se han revelado útiles instrumentos para instalar el Islam sobre otras tradiciones culturales. Las cofradías tienen como centro las zawias. Situadas junto a la tumba de un santo local, las zawias son un importante polo cultural, y con el tiempo se ha convertido también en centro de poder, además de espiritual, político y económico.

\subsection{El muridismo senegalés en inmigración: una estrategia de inser- ción}

Escribía Felice Dassetto a comienzos de los ochenta en relación con su estudio de la influencia Tabligh ${ }^{6}$ entre la población inmigrante marroquí en Bélgica que «las cofradías clásicas funcionan de manera relativamente residual en relación con las sociedad inmigrada y sobre todo con las corrientes hegemónicas del Islam transplantado. En general, los miembros de las cofradías están en relación y organizan reuniones religiosas en casas privadas. No está del todo excluido que en el futuro, en razón incluso de la recomposición en inmigración de las solidaridades segmentarias, las cofradías puedan encontrar bases nuevas de organización y expansión» (F. Dassetto, 1984. 121).

Sin duda, las previsiones, al menos en lo que respecta a la inmigración senegalesa, se habrían cumplido. Y habría que ver hasta qué punto esa recomposición de las solidaridades a través del sistema de cofradías no está influenciada también por la experiencia de la exclusión socioeconómica y las dificultades a la inserción en la sociedad de acogida.

" Según el trabajo de Kepel en Francia, "el Tabligh tiene por objetivo permitir al musulmán, individualmente, reencontrarse con la totalidad de su identidad islámica. Su meta es la reislamización de la sociedad pero, al contrario de los movimientos islamistas; lo hace por abajo, por el individuo. Es una organización de origen indio sin ninguna ambición política, nacida en 1927 en un país en el que los musulmanes eran franca minoría ahogados en el hinduismo. Para el impulsor del tablig en aquel entonces, el objetivo era que los musulmanes indios, en situación de aculturación, vistiendo como hinduistas y celebrando sus fiestas, recuperaran una identidad verdaderamente islámica. En una sociedad no islámica, e donde los signos de pertenencia no son los del Islam y el poder no es islámico, se trata de recrear una estructura comunitaria que rompa con la lógica de la sociedad global, a partir de la reislamización de los individuos" (G. Kepel, en B. López, 1993: 171-172). 
El favorecimiento por parte del Islam de las cofradías de las relaciones comunitarias en base a sus funciones sociales múltiples sería uno de los factores explicativos de su éxito en las condiciones apuntadas: «Estos pueden encontrar en esta forma de Islam un marco míticoritual que les permite explicar e interpretar su experiencia migratoria y reducir las contradicciones inherentes a esta situación. Se asiste en el contexto migratorio a una redefinición de los roles familiares, los roles sexuales, el espacio privado y el espacio público. El carácter religioso de las actividades de grupo no puede más que legitimar las decisiones relativas a las reglas a adoptar. La religión forma parte de esas actividades ritualizadas, donde las reglas fundamentales de una sociedad deben ser observadas con el mayor rigor. La ritualización es más que nunca necesaria aquí para compensar la ausencia de instancias de control de los cambios» (S. Andezian, 1981: 263).

No obstante, advierte Ottavia Schmidt que, si se analiza la pertenencia religiosa del inmigrante senegalés, se corre el peligro de atribuir al murid comportamientos que son propios del senegalés, del inmigrante en general o del individuo. Se corre el peligro de dar un excesivo peso a la pertenencia murid en la definición de la identidad, dejando en la sombra otros aspectos de su personalidad. Pues la noción y la puesta en práctica de la solidaridad comunitaria no es una característica exclusiva de los senegaleses murid. De hecho, la solidaridad y la asistencia acompañan todo el camino del inmigrante senegalés, desde la partida a la llegada, en la vida de todos los días, en las dificultades que éste puede encontrar durante su permanencia lejos de casa, hasta el regreso. Se convierte en una forma de aseguranza contra los riesgos inherentes a la vida precaria del inmigrante (O. Schmidt, 1994: 86).

El sistema de ayuda recíproca se extiende desde la provisión de las condiciones de acogida a la llegada (casa, comida, dinero, trabajo,...) hasta la ayuda para el regreso, pasando por gastos por enfermedad, celebraciones familiares o repatriación de connacionales en caso de fallecimiento.

Afirma Ottavia Schmidt que la fuerza y la originalidad de la emigración senegalesa parece residir en la eficiencia en la organización de la solidaridad, que considera de forma negativa el individualismo. En este sentido, la solidaridad senegalesa no tiene carácter voluntarístico o de emergencia, formaría parte de un estilo y de un sistema de vida que tiene raíces culturales e históricas profundas y que propone valores y modos para realizarlo, como el propio sistema murid (O. Schmidt, 1994: 88-89).

A diferencia de otros grupos de inmigrantes el tipo de ayuda mutua que desarrollan los inmigrantes senegaleses se caracteriza por: 1) el sentido de la jerarquía de edades, 2) la organización y el respeto en el 
cumplimiento de las tareas domésticas y 3) la filosofía del éxito personal, que sólo puede buscarse en el grupo y para el grupo.

Asimismo, los mecanismos comunitarios y de solidaridad senegaleses en inmigración dejan un tanto de lado la influencia de la procedencia étnica. Las diferencias se diluyen en favor de la solidaridad mutua entre connacionales como garantía de supervivencia y de previsión social. La identidad étnica y religiosa, a diferencia del país de origen, no es utilizada en primera instancia en la interacción con la sociedad de acogida (O. Schmidt, 1994).

Por otro lado, el carácter comunitario de la organización de los murid senegaleses, en contraste con otros colectivos inmigrantes, favorece la aparición de intermediarios u operadores sociales que hacen de vínculo entre la comunidad y la sociedad de acogida ${ }^{7}$. El intermediario social suele ser una persona que reside desde hace tiempo en el país de inmigración, conoce la lengua y la cultura y desarrolla una vida social en contacto con la sociedad receptora, actuando como portavoz y como instancia de resolución de conflictos generados entre la comunidad y la sociedad.

De todos modos, y a pesar de su carácter comunitario, el sistema murid da también cabida a múltiples iniciativas individuales. La pertenencia a la cofradía permite a los jóvenes dotados de iniciativa comercial beneficiarse del sistema de solidaridad murid para alcanzar objetivos personales. Es así que el estudio del sistema de solidaridad murid muestra la conjunción de estrategias individuales con otras estrategias más complejas y sistemáticas basadas en la común pertenencia a la cofradía. (O. Schmidt, 1994: 40).

Por ello, puede hablarse también de una estratificación en el interior de la comunidad inmigrante senegalesa. En una primera tipología pueden establecerse tres grupos de murid: los jóvenes rurales que vienen como voto mediante la cadena migratoria, trabajan, envían dinero a casa y a la comunidad y después regresan. Los jóvenes urbanos con un alto nivel de estudios e iniciativa empresarial que pasan a controlar la distribución comercial en relación con la venta ambulante. Los más mayores, que buscan una colocación estable y una reputación, y que mantienen un nivel de interacción con la sociedad de acogida que no les impide mantener los vínculos y la influencia en la comunidad de origen $^{8}$.

7 Hemos podido observar que entre los marroquíes se desarrollan preferentemente estrategias individuales y, en menor grado, grupales, y que por ello no es habitual la figura del intermediario social. Véase J. Lacomba, 1995.

8 Es de este grupo del que surgen los intermediarios sociales, aceptados por su edad y reputación por la comunidad inmigrante y por la sociedad de acogida por su permanencia y conocimiento de la misma. 
Sin embargo, Ottavia Schdmit concluye que no se debe ver en la organización murid sólo una forma particular de solidaridad africana: «se trata de algo más complejo y más original, en donde se entremezclan elementos de la sociedad jerárquica tradicional, de la cultura islámica y del productivismo y consumismo moderno» (O.Schmidt, 1994: 111). El componente económico del sistema murid es fundamental para entender su éxito como estrategia de inserción en una sociedad en la que la religión ocupa una posición residual.

\subsection{Muridismo: el nexo entre economía y religión}

En palabras de Jean Copans «hay entre el muridismo y el desarrollo económico un lazo idéntico al que existe entre el protestantismo y el capitalismo en la hipótesis weberiana» (J. Copans, 1980: 51). Sin entrar a valorar el grado de profundidad de ese paralelismo, no puede sin embargo dejar de señalarse la relación entre determinadas actividades económicas y un particular sistema de creencias y prácticas religiosas en el caso que nos ocupa.

Esas actividades económicas hacen referencia, en primer lugar, y en el caso del país de origen (Senegal), al cultivo del cacahuete (principal producto agrícola para la exportación) tradicionalmente organizado a partir del sistema murid: «La base agronómica, económica y social de la cofradía murid es evidentemente el cacahuete. Ese lazo, que algunos han calificado de fortuito, explica la significación estratégica del trabajo agrícola que produce a la vez el cacahuete y el muridismo» (J. Copans, 1980: 94).

En segundo lugar, y en lo que respecta al ámbito de los países de inmigración, el tipo de actividad económica asociada a la organización murid es el de la venta ambulante. Sin embargo, lo más significativo es que ambos tipos de actividad económica parten en su concepción y organización de una misma filosofía del trabajo, en donde la piedra angular del muridismo resulta ser una división de competencias que se traduce en la delegación al marabut de la plegaria y la meditación, mientras el discípulo se ocupa mediante el trabajo de la prosperidad de la comunidad". La sustitución de la plegaria por el trabajo da de hecho a este último un sentido de redención (O. Schmidt, 1994: 13).

No es casual que haya sido la venta ambulante y no otra la actividad económica desarrollada preferentemente por los inmigrantes senegaleses. Ello por dos razones básicas: la primera es que la venta ambu-

9 Por ejemplo, los Baye Fall (un subgrupo en el interior del muridismo) no cumplen las cinco oraciones, dan gran importancia al trabajo intenso (entremezclando actividad religiosa y actividad comercial), no hacen el Ramadán y no peregrinan a la Meca sino a Tuba. 
lante es uno de los escasos nichos laborales ${ }^{10}$ que el mercado laboral ha dejado sin cubrir en las economías de los países occidentales de inmigración. La segunda, y principal razón, es que la venta ambulante se adapta al carácter informal de las actividades económicas que mayoritariamente realiza la población senegalesa.

«Un sociólogo de Dakar y el equipo Chodak («Paro en Dakar») de ENDA (Medio Ambiente y Desarrollo del Tercer Mundo) se interesaron por el modo de vida de las poblaciones del Gran Yoff, una de las barriadas más desheredadas de Dakar. La estructura reticular permite a unas familias medias de doce personas disponer de un ingreso monetario siete veces superior a los recursos oficiales. El sector informal es objeto del debate, pero los productos de la actividad informal no explican directamente su especificidad; la acumulación de los derechos sobre las diferentes redes en las que participan los miembros de la familia es la fuente directa de la renta complementaria, y no las ventas de bienes y servicios. Una intensa circulación de donativos en dinero, de inversiones, de anticipos, de reembolsos, de contribuciones se ve cubierta por una importante producción de bienes, suministro de servicios, entrega de mercancías. Todo esto se inscribe en unos circuitos complejos: donativos por nacimiento o matrimonio, regalos, empréstitos voluntarios o forzosos, etc. (...) En realidad, el economista no capta más que una parte del aspecto económico de esa realidad compleja pues la mayor parte se halla tan inmersa en la vida social que no es fácilmente discernible» (S. Latouche, 1993: 100).

La venta ambulante, por su carácter informal, permite a los inmigrantes senegaleses mantener una lógica mercantil y comercial que se adapta a la que desarrollan respecto a gran parte de las actividades económicas en su país de origen, al tiempo que les ayuda a no apartarse de su vinculación con la cofradía murid: «Los vendedores ambulantes que distribuyen sus inventarios de baratijas made in HongKong a través de Francia pertenecen en general a la gran familia de la cofradía de los murid. El muridismo senegalés fue fundado en la época colonial por cheik Ahmadou Bamba, para sustraer a los musulmanes del proselitismo de los misioneros cristianos, encuadrándolos estrechamente. Este encuadramiento se reencuentra hoy en día en la organización práctica de la emigración. Los cheiks de las cofradías, propietarios de cultivos de cacahuetes, envían a sus fieles a ganarse la vida de la comunidad en dificultad, puesto que el precio de los productos oleaginosos ha bajado. Los inmigrados navegan entre Dakar y Burdeos, vuelven a la orden del cheik, parten o son reemplazados por novicios.

10 Sobre la cuestión del mercado de trabajo de los inmigrantes en España y los nichos laborales, puede consultarse el artículo de R. Marcos y J. Rojo, 1991. 
Cien mil ambulantes, venidos de Senegal, pertenecen a esta secta, sin contar los clandestinos» (A. Krieger, 1985: 126).

De todos modos, las rémoras asociadas al sistema murid también se han desarrollado en el ámbito de la inmigración: «En Francia, los cheiks han pululado en busca de los vendedores ambulantes para sostenerlos en su presencia espiritual. Incluso allí una alteración se ha producido y muchos de los marabuts son charlatanes que prometen usar su influencia para espantar los malos espíritus. Algunos se dicen capaces de eliminar el mal de ojo, de proporcionar suerte y virilidad, de reconciliar las parejas desunidas (A. Krieger, 1985: 127).

En cuanto al destino de los recursos económicos que el sistema socio-religioso murid genera en inmigración, éste dispone de una doble vertiente. Por un lado, el dinero enviado directamente a los familiares suele ser utilizado en las economías de consumo y en la inversión. Por otro lado, el dinero transferido como donativo a las cofradías (en un porcentaje del que no existen cifras fiables, pero que podría ascender a una cifra significativa) se destina a obras sociales y culturales: cajas de asistencia social, construcción de escuelas, dispensarios médicos, mezquitas u obras públicas (alumbrado, alcantarillado,...).

En la primera dirección la vivienda constituye el sector predilecto de la inversión de los migrantes internacionales senegaleses, con efectos múltiples sobre la sociedad y el tejido urbano: «Los migrantes internacionales, inversores dinámicos en el sector inmobiliario, constituyen una alternativa al desentendimiento del Estado. En efecto, la reducción drástica de las subvenciones estatales a las sociedades inmobiliarias ha sumido a estas últimas en una crisis financiera que les impide realizar sus programas de alojamiento. Los emigrados, que disponen de recursos financieros considerables, parecen haber tomado el relevo. Adquieren de segunda mano unos terrenos en la periferia de la ciudad donde realizan en períodos relativamente cortos construcciones duras. Contribuyen así a la extensión y sobre todo a la revalorización de zonas urbanas y caracterizadas hasta ese momento por su precariedad. Además, en el cercano extrarradio los migrantes internacionales se apropian de casas que transforman para alquilarlas a precios accesibles para los funcionarios y otros empleados del sector informal excluidos de los programas de vivienda planificada" (S.M.Tall, 1994: 137).

Pero es posible establecer también diferenciaciones de acuerdo con la estratificación de la comunidad y las posibilidades económicas de los propios inmigrantes senegaleses: "La localización de las inversiones inmobiliarias está ligada a la vez a la pertenencia étnica, al itinerario migratorio y a la ocupación profesional del emigrado. Los migrantes partidos recientemente construyen casas más modestas, salvo los que se han enriquecido mediante tráficos dudosos o mediante un comercio 
próspero. En las nuevas extensiones de Pikine, encontramos inmigrantes cuya fecha de partida es reciente. La casi totalidad de los inmigrantes encontrados en esta zona se han establecido en los nuevos países de acogida europeos (Italia, España) ${ }^{11}$. Ellos participan en la producción urbana de estos nuevos frentes de urbanización" (S.M. Tall, 1994: 143).

\section{REFERENCIAS BIBLIOGRÁFICAS}

AGUER, B. (1991): «Résurgence de l'Islam en Espagne», en Revue Européenne des Migrations Internationales, vol 7, $\mathrm{n}^{\circ} 3, \mathrm{pp}$. 59-76.

ANDEZIAN, S. (1981): «Appartenance religieuse et appartenance comunautaire: l'exemple d'un groupe d'immigrés algériens en France», en Annuaire de l'Afrique du Nord, pp. 259-266.

BELBAH, M. (1994): «A la recherche des musulmans de France», en G.Kepel. Exils et Royaumes. Paris, PFNSP, pp. 331-345.

BERGER, P. y LUCKMAN, T. (1968): La construcción social de la realidad. Buenos Aires, Amorrortu Editores.

CAMILLERI, C. (1990): Stratégies identitaires. Paris, PUF.

CAMPUS, A. y PERRONE, L. (1990): «Senegalesi et marocchini: inserimento nel mercato del lavoro e progetti migratori a confronto», en Studi Emigrazione, $\mathrm{n}^{\circ} 98$.

CARTER, D. (1991): «Una confraternità mussulmana in emigrazione: i murid del Senegal», en Religioni e Società, ${ }^{\circ} 12$, pp. 60-78.

COMAS, D. y PUJADAS, J. J. (1991): «Familias migrantes: reproducción de la identidad y del sentimiento de pertenencia», en Revista Papers $\mathrm{n}^{\circ} 36$, pp. 33-56.

COPANS, J. (1980): Les marabouts de l'arachide. La confrérie mouride et les paysans du Senegal. Paris, Le Sycomore.

DASSETTO, F. (1988): Le Tabligh en Belgique. Diffuser l'Islam sur les traces du Prophète. Bruxelles, Academia-Sybidi Papers.

DASSETTO, F. (1994): L'Islam in Europa. Torino, Fundazione Giovanni Agnelli.

DASSETTO, F. (1995): «Pratiques sociales et références religieuses», en R. Bistolfi. Islams d'Europe. Intégration ou insertion communautaire?. Paris, Editions de l'Aube.

DASSETTO, F. y BASTENIER, A. (1984): L'Islam Transplanté. Vie et organisations des minorités musulmanes en Belgique. Bruxelles, Evo.

DIOP, M.A. (1989): «Immigration et Religions: Les musulmans négro-africains en France» en Migration et Société, vol.I, 5-6, CIEMI.

1 Inmigrantes senegaleses entrevistados valoran positivamente a España e Italia como países atractivos por la mayor presencia de la economía sumergida e informal y un menor rechazo de la población respecto a otros países europeos. 
DIOP, M.A. (1985): «Les associations Murid en France», en Esprit, $\mathrm{n}^{\circ} 6$, pp. 197-206

DIOP, M.A. (1990): «Un aperçu de l'islam négro-africain en France», en Migrants-Formation $\mathrm{n}^{\circ} 82$, pp. 77-81.

GIMÉNEZ, C. (1995): «Pratiques religieuses, démarches culturelles et insertion socioéconomique», en R.Bistolfi. Islams d'Europe. Paris, Editions de l'Aube, pp. 276-284.

GIRAUD, M. (1993): «Assimilation, pluralisme, double culture: l'ethnicité en question», en R.Gallisot. Pluralisme culturel en Europe: cultures européennes et cultures des diasporas. Paris, L'Harmattan, pp. 233-246.

KEPEL, G. (1987): Les Banlieues de l'Islam. Paris, Seuil.

KEPEL, G. (1993): «El Islam en Francia. ¿Inserción o integración?», en B. López. Inmigración magrebi en España. El retorno de los moriscos. Madrid, Mapfre, pp. 161-180.

KRIEGER, A. (1985): Les musulmans en France. Religion et culture. Paris, Maisonneuve et Larose.

LACOMBA, J. y ROYO, I. (1995): Estudio de las condiciones de vida de los inmigrantes marroquies y senegaleses en situación irregular en el área urbana de Valencia. Memoria de investigación. Institución Valenciana de Estudios e Investigaciones.

LACOMBA, J. (1996): Los factores culturales en la inserción de los colectivos migrantes. El caso de la inmigración musulmana. Cuadernos Citmi $\mathrm{n}^{\circ} 1$, pp. 59-68.

LATOUCHE, S. (1993): El planeta de los naufragos. Madrid, Acento Editorial.

LEVEAU, R. (1988): Les Musulmans dans la Société Française. Paris, PFNSP.

LEWIS, B. y SCHNAPPER, D. (1992): Musulmans en Europe. Poitiers, Actes Sud.

MARCOS, R. de y ROJO, J. (1991): «Trabajadores extranjeros en España» en Revista Economía y Sociología del Trabajo, n ${ }^{\circ} 11$, pp. 8-17.

MARTINIELLO, M. (1994): «Elites et leaders ethniques: entre mobilité sociale et structuration communautaire», en Revue Internationale d'Action Communautaire $\mathrm{n}^{\circ} 31 / 71$, pp. 105-117.

PEROTTI, A. (1994): «L'Immigration et le fait religieux», en Revue MigrationsSociété, vol 6, n³3-34, pp. 5-8.

REYSSO, F. (1991): Pélerinages au Maroc. Paris, Editions de la Maison de Science de l'Homme.

SCHMIDT, O. (1994): Islam, solidarietá e lavoro. I muridi senegalesi in Italia. Fondazione Giovanni Agnelli, Torino.

SCHNAPPER, D. (1988): «Modernidad y aculturaciones a propósito de los trabajadores emigrantes», en T.Todorov. Cruce de culturas y mestizaje cultural. Gijón, Júcar, pp. 173-205. 
TALL, M.S. (1994): «Les investissements immobiliers à Dakar des émigrants sénégalais», en Revue Européenne des Migrations Internationales. Volum 10, n³, pp. 137-151.

WERNER, J.F. (1993): Marges, sexes et drogues à Dakar. Paris, Karthala. 